Article

\title{
Detection of Parkinson's Disease from 3T T1 Weighted MRI Scans Using 3D Convolutional Neural Network
}

\author{
Sabyasachi Chakraborty ${ }^{1}$ (D), Satyabrata Aich ${ }^{2, *}$ (i) and Hee-Cheol Kim ${ }^{2,3, *}$ \\ 1 Department of Computer Engineering, Inje University, Gimhae 50834, Korea; c.sabyasachi99@gmail.com \\ 2 Institute of Digital Anti-Aging Healthcare, Inje University, Gimhae 50834, Korea \\ 3 Ubiquitous Healthcare \& Anti-aging Research Center (u-HARC), Inje University, Gimhae 50834, Korea \\ * Correspondence: satyabrataaich@gmail.com (S.A.); heeki@inje.ac.kr (H.-C.K.); \\ Tel.: +82-55-320-3720 (S.A. \& H.-C.K.)
}

Received: 14 May 2020; Accepted: 10 June 2020; Published: 12 June 2020

check for updates

\begin{abstract}
Parkinson's Disease is a neurodegenerative disease that affects the aging population and is caused by a progressive loss of dopaminergic neurons in the substantia nigra pars compacta (SNc). With the onset of the disease, the patients suffer from mobility disorders such as tremors, bradykinesia, impairment of posture and balance, etc., and it progressively worsens in the due course of time. Additionally, as there is an exponential growth of the aging population in the world the number of people suffering from Parkinson's Disease is increasing and it levies a huge economic burden on governments. However, until now no therapeutic method has been discovered for completely eradicating the disease from a person's body after it's onset. Therefore, the early detection of Parkinson's Disease is of paramount importance to tackle the progressive loss of dopaminergic neurons in patients to serve them with a better life. In this study, 3T T1-weighted MRI scans were acquired from the Parkinson's Progression Markers Initiative (PPMI) database of 406 subjects from baseline visit, where 203 were healthy and 203 were suffering from Parkinson's Disease. Following data pre-processing, a 3D convolutional neural network (CNN) architecture was developed for learning the intricate patterns in the Magnetic Resonance Imaging (MRI) scans for the detection of Parkinson's Disease. In the end, it was observed that the developed 3D CNN model performed superiorly by completely aligning with the hypothesis of the study and plotted an overall accuracy of $95.29 \%$, average recall of 0.943 , average precision of 0.927 , average specificity of 0.9430 , f1-score of 0.936, and Receiver Operating Characteristic-Area Under Curve (ROC-AUC) score of 0.98 for both the classes respectively.
\end{abstract}

Keywords: Parkinson's Disease; neurodegeneration; magnetic resonance imaging (MRI); convolutional neural network (CNN); deep learning

\section{Introduction}

The second most common neurological disorder that prevails among the aging population in the world is considered to be Parkinson's Disease (PD). Parkinson's Disease primarily affects the nerve cells in the brain that are responsible for producing dopamine, which is an organic chemical that acts as a neurotransmitter to transmit signals between nerve cells. Therefore, patients having Parkinson's Disease usually suffer from cognitive and movement disorders such as muscle stiffness, tremors, and impairment of posture and balance. Moreover, Parkinson's Disease is progressive in nature, therefore, early detection and monitoring of the disease leads to improvement in the life of the patients. Also, as the aging population across the globe in increasing exponentially, a requirement for 
the development of suitable methods for the detection of Parkinson's Disease at a very early stage is indeed very important [1-5].

For the early detection of Parkinson's Disease, the most widely used diagnostic paradigm is the analysis of Magnetic Resonance Imaging (MRI) scans of the brain. The MRI scans provide anatomical details about the subcortical structures of the brain that are further analyzed to check for any aneurysms, which further deems helpful for the early diagnosis of a particular type of disease. However, as the MRI is a 3D structure, it becomes really difficult for the human eye to analyze the intrinsic details and heterogeneous properties of subcortical structures [6,7]. Therefore, with the advancement of intelligent technologies, computer-aided detection systems have proven to be very effective in performing analysis and diagnosis of diseases by leveraging multi-dimensional healthcare data.

In the past researchers performed multiple studies and found out that the textural and morphological analysis of the tissue and cell imaging scans have provided some very astonishing results. The application of textural and morphological analysis was considered to be huge as it was able to perform the quantification of grey level patterns and derive the inter-pixel relationship within the regions of interest. Moreover, it was also observed that different areas in a scan or an image had different textural and morphological patterns which were difficult for human beings to track [8]. Therefore, textural and morphological analysis of the imaging scans proved to be very much reliable for neurological studies and applications in the detection and diagnosis of diseases. But with the advances in the field of computer applications and intelligent systems, the research community is now focusing more on data-driven feature representation rather than handcrafted feature engineering which requires domain-specific knowledge [9]. Therefore, with the rapid development of deep learning architectures and technologies, it is proving to lay down some state-of-art methodologies for medical image applications.

The paper is structured as follows. The related works in the field of the detection of Parkinson's Disease and other Neurodegenerative diseases using deep learning and machine learning algorithms are described in Section 2. Section 3 discusses the data used in the study specifically, the data selection procedure, imaging modalities, and image registration protocols. Moreover, the section demonstrates the complete workflow for the processing of the Parkinson's Progression Markers Initiative (PPMI) data and registering the data with respect to MNIPD25-T1MPRAGE-1 mm atlas. Section 4 underlines the methodology of the study concerning the development and optimization of the 3D convolutional neural network architecture. Section 5 discusses the performance and generalizing capability of the developed 3D convolutional neural network architecture. Section 6 further discusses the whole study and discusses the importance of the work for the early detection of Parkinson's Disease. Finally, the paper is concluded in Section 7 and the future work regarding the study is discussed.

\section{Related Work}

In recent years the use of deep learning for image analysis has presented some of the most astounding results. More advertently in the field of medical image analysis such as classification, segmentation, etc. [10-12], convolutional neural networks (CNN) [13] had been considered to demonstrate some state-of-the-art results and also are used in many real-world applications. In the field of Alzheimer's disease (AD) detection, multiple notable works have been performed and presented some state-of-the-art results. Payan and Montana [14] performed a study where 2265 historical MRI scans were obtained from the Alzheimer's Disease Neuroimaging Initiative (ADNI) and further all the MRI scans were subjected to a sparse-autoencoder followed by a 3D CNN network. The classification of AD vs. healthy control (HC) vs. mild cognitive impairment (MCI) was plotted at an accuracy of $89.47 \%$. Also, Hosseini et al. [15] undertook a study for the classification of AD, HC, and MCI by leveraging 3D convolutional autoencoder on normalized T1 weighted MRI scans from the ADNI database. Similarly, others have also demonstrated their works where CNN was leveraged in MRI and Functional magnetic resonance imaging (fMRI) scans for the detection of $\mathrm{AD}$ and the classification of MCI and HC [16-20]. 
On the other hand, for the early detection of Parkinson's Disease, multiple works have been performed considering the texture and morphological analysis of the region of interest (ROI) and voxels of interest (VOI) of subcortical structures of the brain. Li et al. [21] performed a study regarding the 3D texture analysis of the substantia nigra using the quantitative susceptibility maps (QSM) and R2* maps for the detection of Parkinson's Disease. Further, the study extracted first- and second-order textural features from QSM and R2* maps and it was found that first- and the second-order QSM maps accurately distinguished PD from HC. Similarly, Sikio et al. [22] proposed a technique to determine the structural changes of the brain from an MRI baseline using textural features. It was observed from the study that the textural features can be considered for discriminating the structural changes. Moreover, similar studies were also performed by $[4,23]$ where the textural and morphological features were only considered from specific subcortical structures of the brain for the prediction of Parkinson's Disease. But concerning the implementation of $\mathrm{CNN}$ and deep learning on MRI scans for the detection of Parkinson's Disease, there are quite a few notable works that have been performed. Therefore, following in this section some related studies are discussed that have been considered to demonstrate state of the art results.

Ortiz et al. [24] performed a study for the detection of Parkinson's Disease using features based on the isosurface of 3D brain Single-Photon Emission Computed Tomography (SPECT) scans. For the study, the authors acquired the DaTscan SPECT scans from PPMI database. The SPECT scans were further subjected to a feature extraction method that extracted only the isosurface or isolines (2D version of isosurfaces) from the 3D SPECT scans. Further, the isosurfaces were subjected to a 3D CNN model based on the characteristics of AlexNet and it was observed that the model plotted a specificity and sensitivity of $95 \%$ and receiver operating characteristic (ROC) of 0.97 . Similarly, Kollia et al. [25] also performed an interesting study where they predicted Parkinson's Disease from MRI and DaT scan data by leveraging latent information from deep convolutional neural networks. In the study, the authors implemented two deep neural networks, one with the normal specification of convolution layers followed by fully connected layers and the other one where the normal architecture was followed by a recurrent layer of gated recurrent unit (GRU) neurons. Further, latent variables were extracted from the fully connected layer of the trained CNN models and multiple approaches for the detection were performed. The approaches included combinations of transfer learning, clustering [26], and nearest neighbors for enhancing the classification performance of the model.

Shinde et al. [27] in his work regarding the detection of Parkinson's Disease leveraged neuromelanin sensitive MRI (NMS-MRI), which can identify abnormalities in the substantia nigra (SNc) in Parkinson's Disease patients. Moreover, the author also plotted concerns over the handcrafted features based on the contrast ratio, area, and volumes of the subcortical structures [28-31]. Further, the authors employed a CNN network for the detection of PD from HC and demonstrated optimum test accuracy of $80 \%$ concerning ratio-based and radiomics feature-based classification techniques. In another study, Sivaranjini et al. [32] leveraged AlexNet [33] architecture on MRI images obtained from the PPMI database for the detection of Parkinson's Disease. The model provided an optimum accuracy of $88.9 \%$ while distinguishing PD from HC. Similar studies were further performed by [34-36] by leveraging multiple imaging modalities, multiple imaging cohorts, and different types of deep learning architecture. The studies further performed superiorly in identifying Parkinson's Disease from healthy control.

The aforementioned literature further proves the diligence of the analysis of MRI by leveraging deep learning architectures for the detection of Parkinson's Disease. Moreover, it has also been established that deep learning architectures and more specifically convolutional neural networks perform superiorly in extracting hierarchical information from imaging modalities. Therefore, the findings plotted by the aforementioned research studies motivated us to perform an analysis of the MRI scans using a 3D $\mathrm{CNN}$ network for the detection of Parkinson's Disease. 


\section{Data Collection and Preprocessing}

\subsection{Data Collection}

The data for the study was collected from the PPMI database (www.ppmi-info.org/data). PPMI database for neuroimages is considered to be a landmark, international, and multicenter study to research the biomarker's that are responsible for Parkinson's Disease progression. The MRI scans selected for the study were based on particular imaging protocols described in Table 1 and also corresponds to the baseline visit. Further, all the scans that were considered in the study were obtained from a single type of scanner, i.e., Siemens, Munich, Germany. Moreover, all the acquired scans were based on Magnetization Prepared-Rapid Gradient Echo (MP-RAGE) sequence. All the scans used in the study were acquired in a time range of 20-30 min field of view (FoV) of all the scans including vertex, cerebellum, and pons.

Table 1. Imaging protocol for choosing the scans from Parkinson's Progression Markers Initiative (PPMI) database.

\begin{tabular}{cc}
\hline Imaging Protocol & Values \\
\hline Modality & Magnetic Resonance Imaging (MRI) \\
Research Group & Control and Parkinson's Disease (PD) \\
Visit & Baseline \\
Acquisition Plane & Sagittal \\
Acquisition Type & $3 \mathrm{D}$ \\
Field Strength & 3.0 Tesla \\
Flip Angle & 9 Degree \\
Scanner Manufacturing & Siemens Magnetization Prepared-Rapid Gradient \\
Pixel Spacing & Echo (MPRAGE) \\
Slice Thickness & $0.9-1.5$ mm (X \&Y) \\
Weighting & $1.0 \mathrm{~mm}$ \\
& $\mathrm{T1}$ \\
\hline
\end{tabular}

Post applying the filter based on the imaging protocol mentioned in Table 1, a total of $406 \mathrm{MRI}$ scans were selected from the baseline visit of the patients. Out of 406 patients, 148 were female and 258 were male. The scans that were considered for the study belonged to the subjects aged 62.64 \pm 9.9 . The scans primarily belonged to two research groups that are healthy control (HC) and Parkinson's Disease (PD). The scans were distributed into the respective research groups as 203 for healthy control and 203 for Parkinson's Disease. The subjects who were considered for obtaining the scan were selected on certain criteria described in Table 2.

Table 2. Eligibility criteria for the subject to be included in a research group.

\begin{tabular}{|c|c|}
\hline Research Group & Criteria \\
\hline Parkinson's Disease & $\begin{array}{l}\text { - } \quad \text { Patients must have at least two: resting tremor, bradykinesia, rigidity, } \\
\text { asymmetric resting tremor, asymmetric bradykinesia. } \\
\text { - } \quad \text { Diagnosis of Parkinson's Disease for two years. } \\
\text { - } \quad \text { Hoehn and Yahr stage I or II. } \\
\text { - } \quad \text { Male or Female age } 30 \text { Years or Older. }\end{array}$ \\
\hline Control Subjects & $\begin{array}{l}\text { - Male or Female age } 30 \text { Years or Older. } \\
\text { - Not First degree relative to Parkinson's patient. }\end{array}$ \\
\hline
\end{tabular}

Table 3 shows the specifications of the scans that were obtained from the PPMI database. While Figure 1 depicted shows the sample of MRI scans belonging to both the research groups that were obtained from the PPMI database. 
Table 3. Specification of the acquired scans from PPMI.

\begin{tabular}{cc}
\hline Image Parameters & Values \\
\hline Dimensions & $256 \times 256 \times 170-200$ pixels \\
Interslice Gap & $1.0 \mathrm{~mm}$ \\
Slice Thickness & $1.0 \mathrm{~mm}$ \\
Spacing & $1.0 \times 1.0 \times 1.0 \mathrm{~mm}$ \\
Plane & Sagittal \\
\hline
\end{tabular}

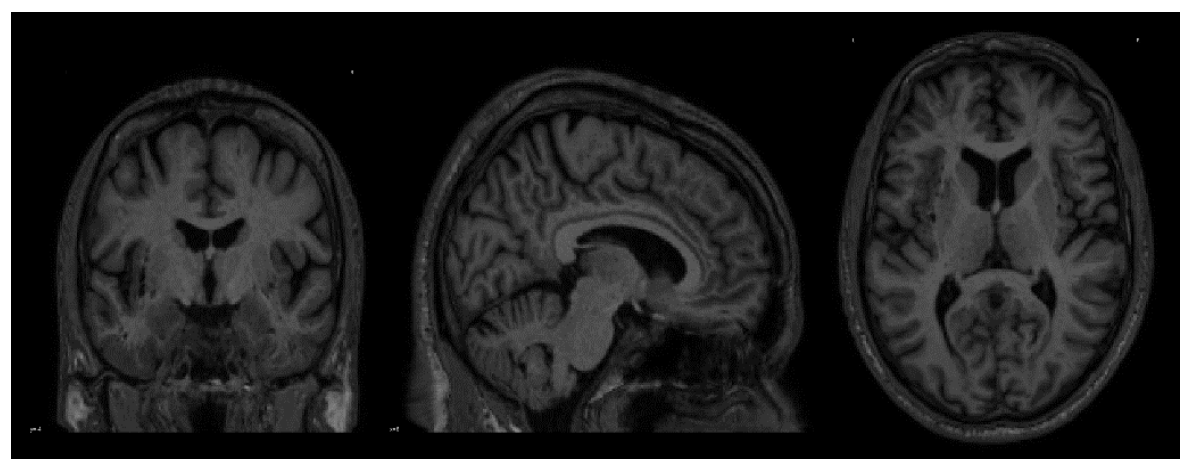

Coronal

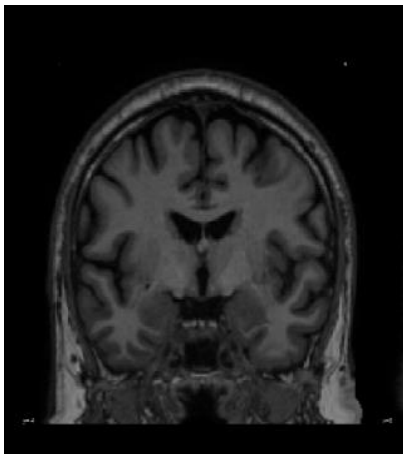

Coronal
Sagittal

(a)
Axial

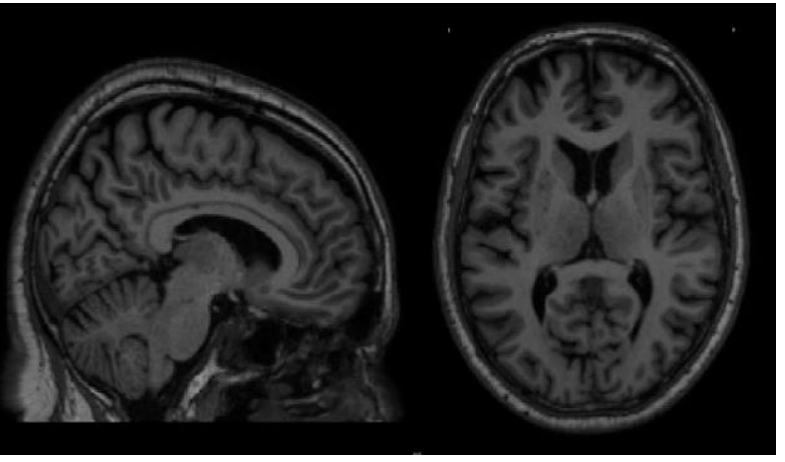

Sagittal

Axial

(b)

Figure 1. Sample of Magnetic Resonance Imaging (MRI) scans obtained from the Parkinson's Progression Markers Initiative (PPMI) database (a) MRI scan of a subject from the control group; (b) MRI scan of a subject from to the Parkinson's Disease group.

\subsection{Data Preprocessing}

The dataset that was used in the study was obtained from the PPMI database; as previously mentioned, PPMI is a multicenter study, therefore, the imaging scans acquired in the study contained temporal and spatial differences. To solve this particular problem and to maintain a constant modality between all the scans it was required that all the scans needed to be in the same space such as Montreal Neurological Institute (MNI) [37,38] or individual brain atlases using statistical parametric mapping (IBASPM) [39]. Therefore, to transform the PPMI MRI data that has been collected from multiple centers across the globe to a fixed coordinate system, an image registration procedure was performed. Image registration is a process where traversal is performed on a fixed image (atlas) to find the alignment parameters and coordinates so that an unknown or an unseen image can be aligned similarly to the fixed image. Trivially, image registration could be understood as the process of aligning two images to a particular space where one acts as the source image and the other as target image, and the source image is transformed in a method to align with the target image. In the specific study, the MRI scans 
obtained from the PPMI database were considered as the source image and the atlas, such as MNI or IBASPM, were considered as the target image.

The registration of the MRI scans obtained from the PPMI database was performed using MNIPD25-T1MPRAGE-1 $\mathrm{mm}$ atlas created by [40-42]. The specifications of the MNIPD25-T1MPRAGE-1 mm atlas is described in Table 4. The registration of the MRI scans was performed using one of the most effective normalization tools known as advanced normalization tools python (ANTsPy) [43]. ANTsPy is used particularly in the field of imaging research for extracting important information from complex imaging datasets to perform preprocessing on MRI, fMRI, and SPECT data. The registration of the acquired MRI scans with the MNIPD25-T1MPRAGE-1 mm atlas was performed using symmetric normalization. Figure 2 depicts a particular MRI scan before and after the registration process.

Table 4. Specification of MNIPD25-T1MPRAGE-1 mm atlas.

\begin{tabular}{cc}
\hline Image Parameters & Values \\
\hline Dimensions & $193 \times 229 \times 193$ pixels \\
Interslice Gap & $0.0 \mathrm{~mm}$ \\
Slice Thickness & $1.0 \mathrm{~mm}$ \\
Spacing & $1.0 \times 1.0 \times 1.0 \mathrm{~mm}$ \\
Plane & Sagittal \\
\hline
\end{tabular}

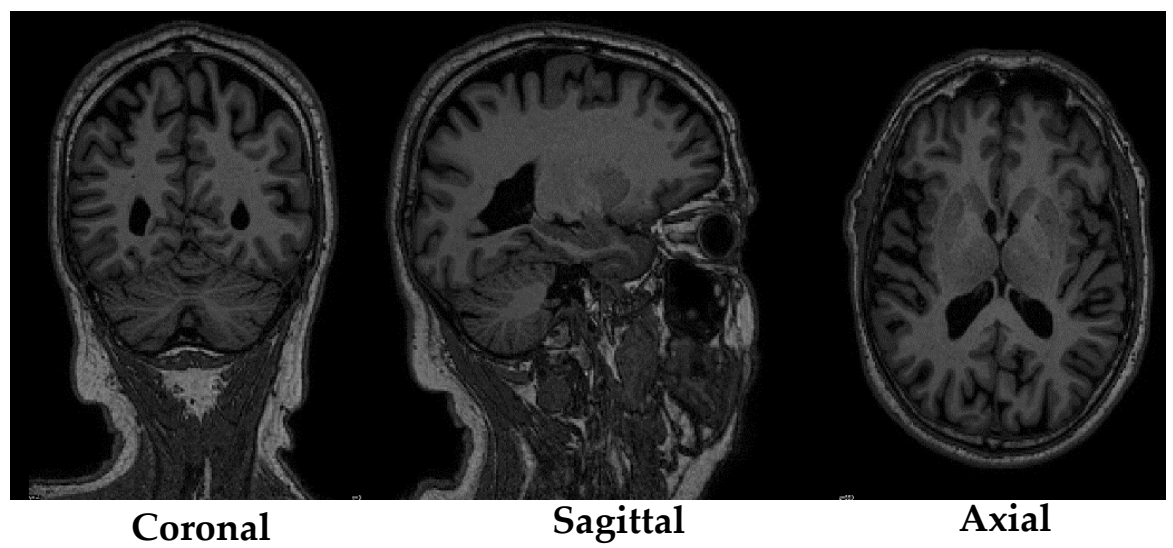

(a)

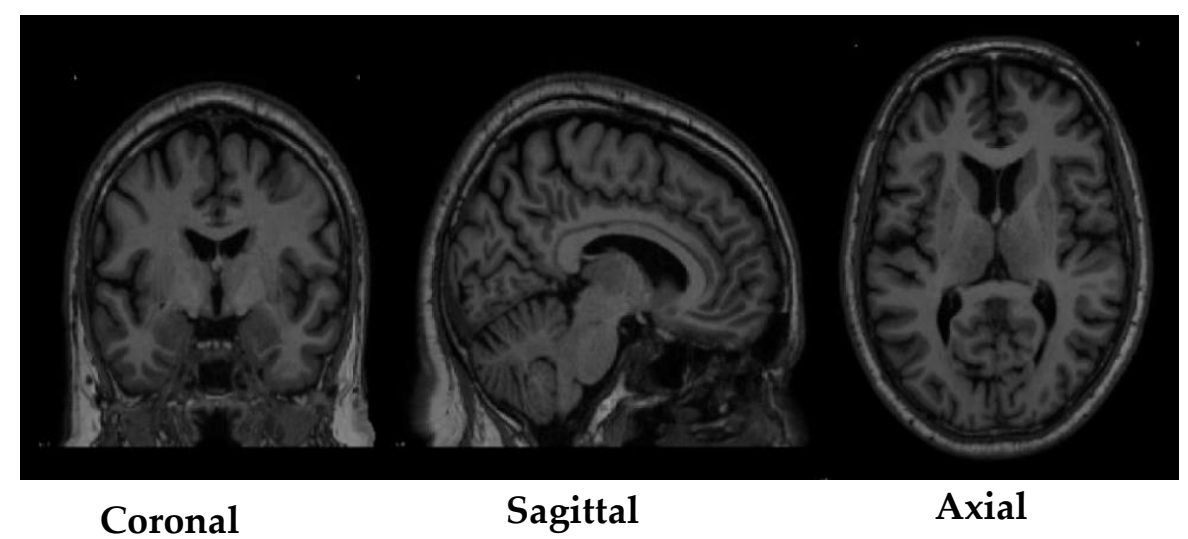

(b)

Figure 2. Before and after registration of a particular scan, (a) MRI scan before registration and (b) MRI scan after registration. 


\section{Materials and Methods}

The main premise of the study focusses on the detection of Parkinson's Disease and the classification of MRI scans as healthy control or Parkinson's Disease using 3D convolutional neural networks. The complete flow of process and methodology for the detection of Parkinson's Disease is described in Figure 3. The methodology was primarily divided in to four stages: MRI scan acquisition from the PPMI database; data preprocessing, registration, and transformation; 3D convolutional neural network architecture; and finally the results and performance evaluation of the CNN architecture based on some metrices. The first two stages of the methodology have been thoroughly discussed in Section 3 and the third and the fourth stage will be discussed in the following sections.

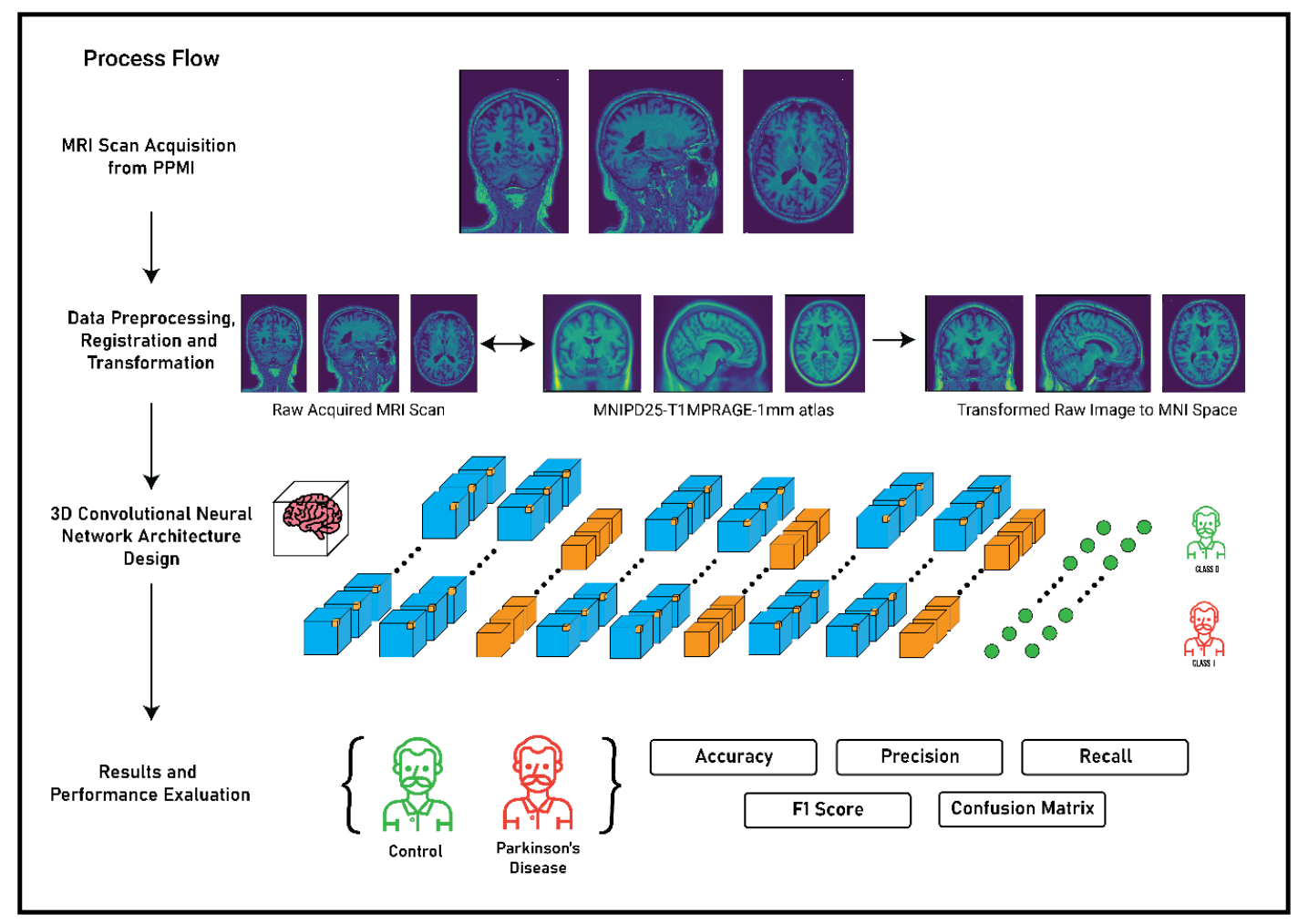

Figure 3. Complete process flow of the study.

\subsection{D Convolutional Neural Network Architecture}

In recent times, supervised learning techniques for solving problems have evolved massively. Moreover, the popularity and effectiveness of deep learning algorithms have also undergone a major paradigm shift in terms of architectural designs and optimizer functions [43]. Particularly, in the field of health care, deep learning algorithms have shown much predominance over the previous techniques that were used for imaging analysis, aneurysms detection in images, biosignal analysis, etc.

In this paper, a 3D convolutional neural network model has been developed for the detection of Parkinson's Disease from T1 weighted MRI scans. The primary proposition of the work presents a system that can be used to identify Parkinson's Disease from MRI or brain images. Additionally, the second proposition of the study was to determine the plausible regions of interest (ROIs) in the brain MRI images that are responsible for Parkinson's Disease. Therefore, to solve the primary proposition of the study a 3D convolutional neural network has been developed as shown in Figure 4 and Table 5 . The CNN network developed in the work consists of 35 layers including the input and the output layer. Further, the network architecture consists of 12 3D convolution layers, which allows the model to create the feature representations of the input brain MRI scans. Moreover, all the convolution layers are supported by activation functions. Further, all the feature representations are subjected to max-pooling 
layers which are responsible for down-sampling the input feature matrix and provides an abstract form of the feature representation to avoid overfitting. After the complete process of feature learning, all the feature matrices are flattened so that it can be accepted by the dense layer or the fully connected layer. The representations from the dense layer are further subjected to the output dense layer with two neurons and sigmoid activation which corresponds to the two states that are healthy control and Parkinson's Disease.

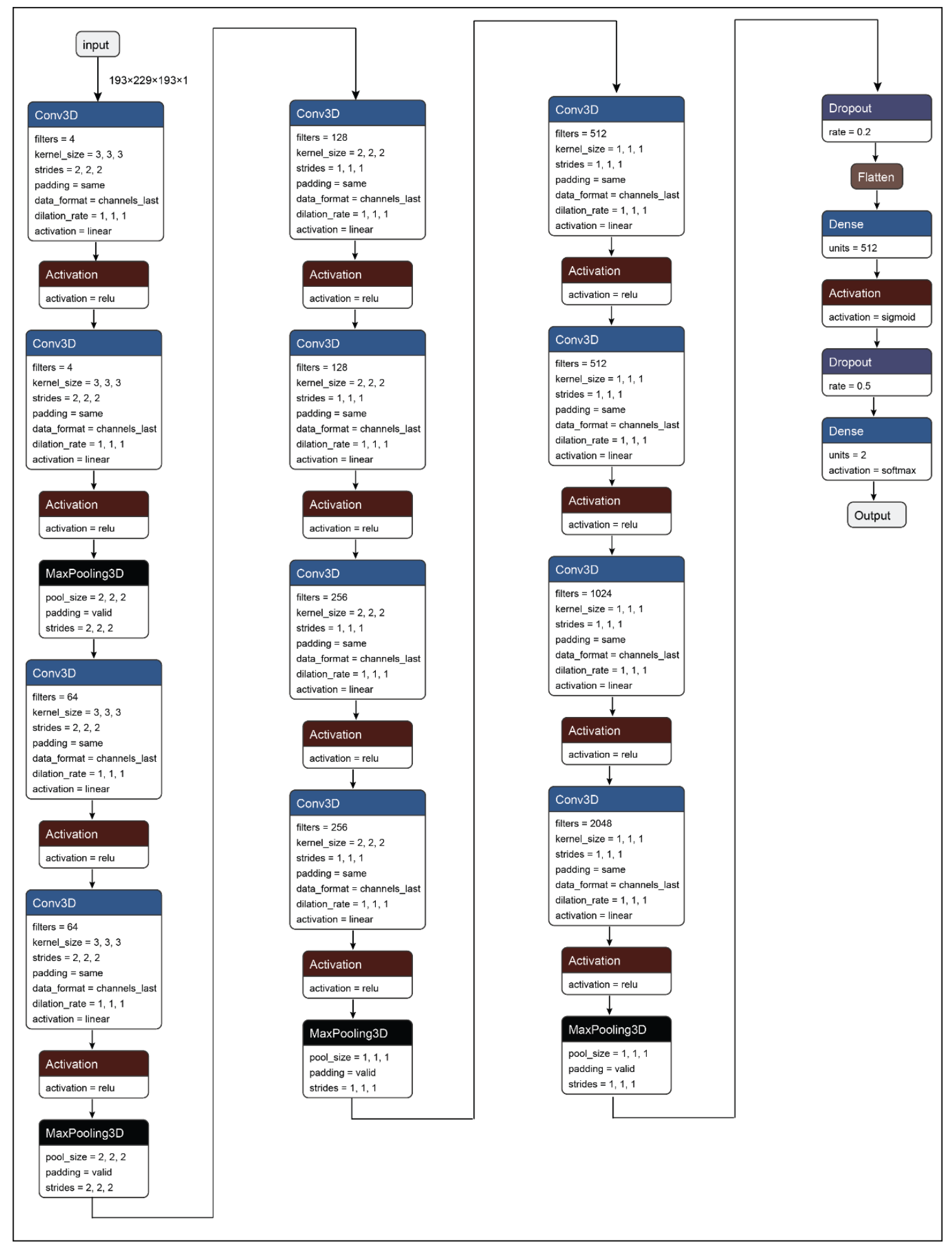

Figure 4. 3D Convolutional Neural Network (CNN) architecture. 
Table 5. 3D convolutional neural network (CNN) architecture.

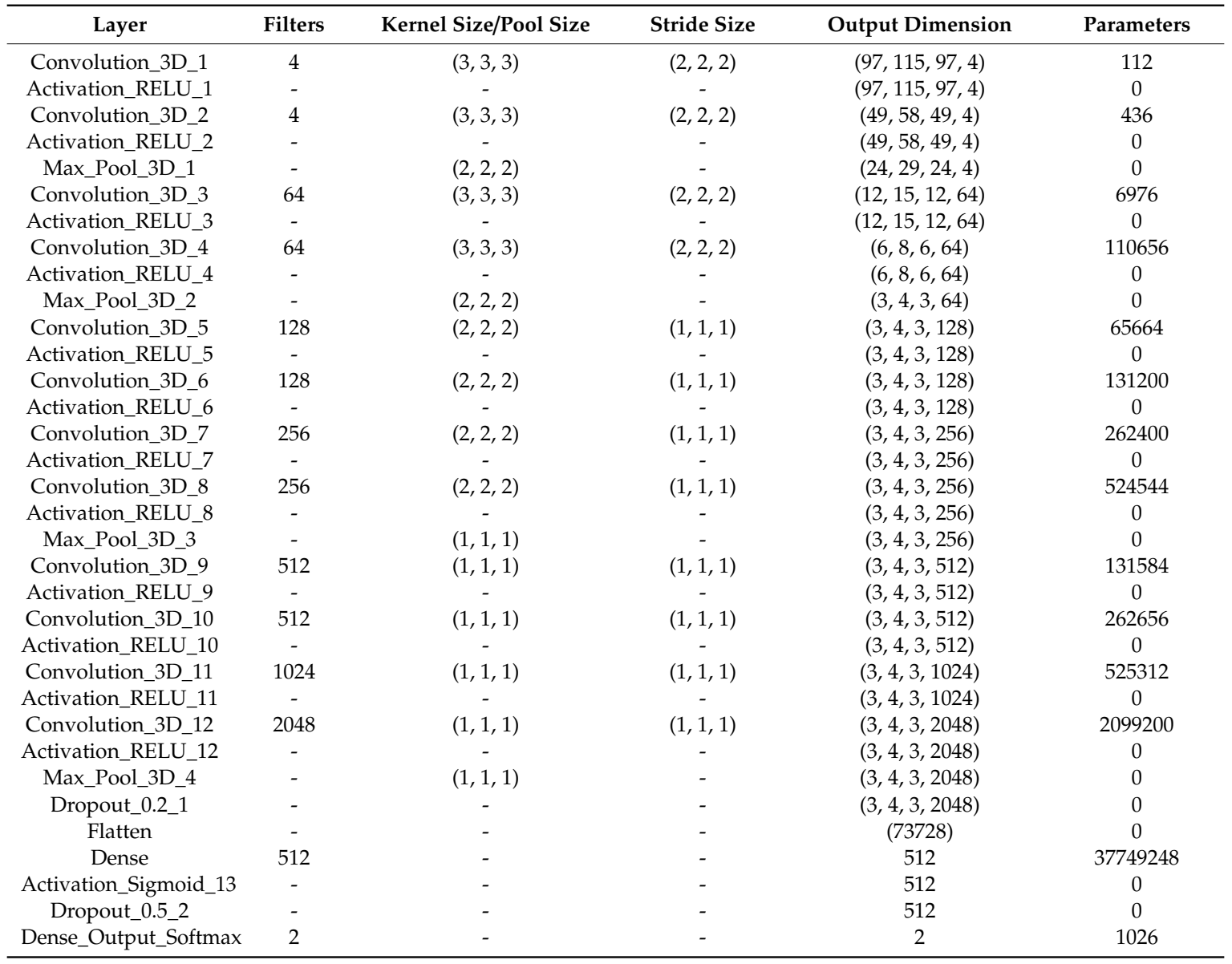

\subsection{Hypothesis and Training Procedure}

For developing the statistical, machine learning, and deep learning model, the first step is considered to be the development of the hypothesis of the problem that needs to be solved. Therefore, the primary hypothesis that was devised for solving a particular problem is as follows:

1. The recall of the Parkinson's Disease class or the true negatives must be more than 0.95 and the probability factor of the mispredicted samples or the false negatives must not be more than $60 \%$.

2. The recall of the healthy class or the true positives must be more than $85 \%$ and the probability factor of the mispredicted samples or the false positives must not be more than $65 \%$.

Therefore, based on the above hypotheses, the performance of the 3D convolutional neural network model was evaluated. For the evaluation purpose, five different classification performance metrics were considered namely accuracy, precision, recall, f1-score, and confusion matrix. Also, for determining the generalizability of the model over unseen data a 5-split cross-validation was performed. The details regarding the evaluation of the performance metrics are described in the Results section.

\subsection{Model Optimizer Hyperparameters and Loss}

The development of the 3D CNN architecture is indeed the most important aspect of the work. However, the component that needs to be considered carefully for creating the learning algorithm is choosing the right set of hyperparameters for optimizing the internal set of parameters of the network such as weights and biases, and the loss function. 
The process of controlling the training process is considered very important while creating a deep learning model. The process is undertaken by the hyperparameters of the optimizer function that is responsible for tuning the optimizer algorithms. For the present study, the primary aspect that lies in the optimization algorithm is to minimize the validation and the testing error of the model. For performing the specific task, the hyperparameters that reside outside the primary deep learning model must be tuned in such a way we generate the perfect internal parameters of the model that are the weights and biases. However, the challenge that is faced in the process is that the hyperparameters much be chosen in a particular way that it should be model-specific rather than a training set to increase the generalizability of the model over unseen data. Therefore, for choosing the perfect set of hyperparameters to maintain the overall model generalizability and optimum objective score, Bayesian sequential model-based optimization (SMBO) is used.

Bayesian SMBO is an algorithm used for hyperparameter optimization the works to minimize an objective function by creating a surrogate model (probability function) based on the evaluation results of the previous objective function. The basic objective function of the Bayesian SMBO is given as:

$$
P(\text { score } \mid \text { hyperparameters })=\frac{P(\text { hyperparameters } \mid \text { score }) P(\text { score })}{P(\text { hyperparameters })}
$$

The surrogate model that is developed by the Bayesian SMBO is considered to be less expensive than the main optimizer function [44]. Further, the next set of evaluation results are selected by using the expected improvement criterion [45]. The criterion is defined as:

$$
E I(x)=E\left(\max \left(f(x)-f^{*}, 0\right)\right)
$$

where $x$ belongs to the hyperparameter values and is considered to be an improvement in the objective sore of $f(x)$, and $f^{*}$ is the maximum value of the objective score found in the process.

Further, in the process, AdaDelta [46] is chosen as the optimizer algorithm for optimizing the weights and biases of the network. AdaDelta is considered to be a very robust algorithm relating to the gradient descent algorithm. The algorithm dynamically adapts over the course of the training process by leveraging only first-order information. Moreover, the algorithm does not require any manual tuning of the learning rate and is very robust towards noisy gradient information. Therefore, Bayesian $\mathrm{SMBO}$ was applied to the algorithm to generate the optimum hyperparameters and is mentioned below.

Learning rate: 0.08423 ; rho: 0.625 ; epsilon: 1.0

Another very integral part of the deep learning models is the loss functions. These functions are typically used to determine the variability between the prediction $(\hat{y})$ and the true value $(y)$. The output of the loss functions is a non-negative value that increases the generalizability of the model by decreasing the loss [47]. The loss function of a model is given by:

$$
L(\theta)=\frac{1}{n} \sum_{i=1}^{n} L\left(y^{(i)}, f\left(x^{(i)}, \theta\right)\right.
$$

where $\theta$ represents the parameters of the model, $x$ represents the feature matrix, and $y$ represents the true values for a particular set of features.

The loss function used in the present study was binary cross-entropy, which is also known as the cross-entropy or the log loss. In the binary cross-entropy loss, each prediction outcome is compared to the true value and a loss score is calculated. The loss score in the process is used to penalize the probability of the prediction. The loss score is logarithmic which means a small penalty is allotted to tiny differences between prediction outcome and true value and a large penalty is applied to bigger 
differences [48]. The equation of binary cross-entropy is given where $y$ is the true value and $p(y)$ is the predicted probability of $y$ being true.

$$
H_{p}(q)=-\frac{1}{N} \sum_{i=1}^{N} y_{i} \log \left(p\left(y_{i}\right)\right)+\left(1-y_{i}\right) \log \left(1-p\left(y_{i}\right)\right)
$$

\section{Results}

The 3D convolutional neural network model presented decent results in terms of detecting Parkinson's Disease from brain MRI scans. The model quantitatively presented effective results by prompting an average recall and precision of 0.9421 and 0.9280 for both the classes, respectively. Also, for the training procedure, a 5-split cross-validation with the ratio of 80:20 was performed over the complete dataset, and it was observed that the model demonstrated optimum generalizability in terms of predicting the data according to multiple cross-validation test sets. Table 6 shows the results of the 5 -split cross-validation that was used to determine the generalizability of the 3D convolutional neural network model over unseen data.

Table 6. Performance evaluation of the 3D CNN model.

\begin{tabular}{llllll}
\hline Metrics & Split 1 & Split 2 & Split 3 & Split 4 & Split 5 \\
\hline Accuracy & $93.24 \%$ & $90.21 \%$ & $92.68 \%$ & $95.29 \%$ & $92.33 \%$ \\
Precision & 0.9270 & 0.9340 & 0.9520 & 0.9270 & 0.9100 \\
Specificity & 0.9112 & 0.8843 & 0.9100 & 0.90 & 0.9263 \\
Recall & 0.9140 & 0.9157 & 0.9310 & 0.9430 & 0.9240 \\
F1-Score & 0.9366 & 0.9290 & 0.9428 & 0.9360 & 0.9160 \\
ROC-AUC & 0.94 & 0.92 & 0.95 & 0.98 & 0.94 \\
\hline
\end{tabular}

Figure 5 depicts the confusion matrix that was generated based upon the results received from the best performing cross-validation set. Also, it can be observed that the confusion matrix completely aligns with the prior hypothesis which states that there must not be any misprediction of the MRI scans belonging to the PD class into any other class and the recall of prediction in the healthy class must be more than $85 \%$. However, analyzing just the predictive performance of the deep learning models are not sufficient to determine the credibility of the model. Therefore, in Figures 6 and 7 quantile-quantile plots $(\mathrm{QQ})$ have been depicted to understand the uncertainty of the model and the confidence of predictions.

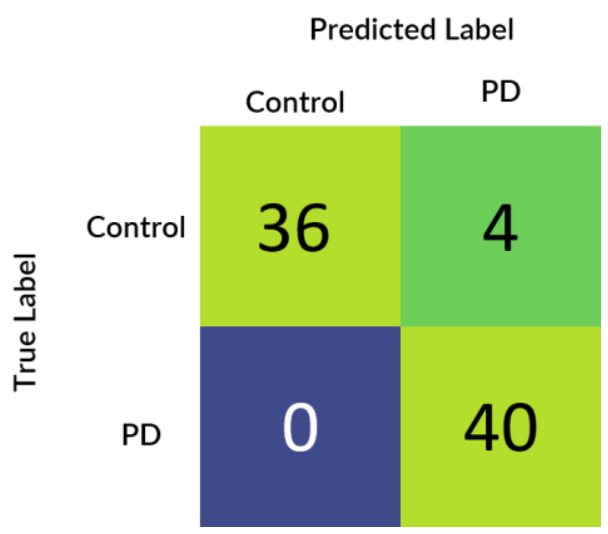

Figure 5. Confusion matrix of the 4th cross-validation split, where PD stands for Parkinson's Disease. 


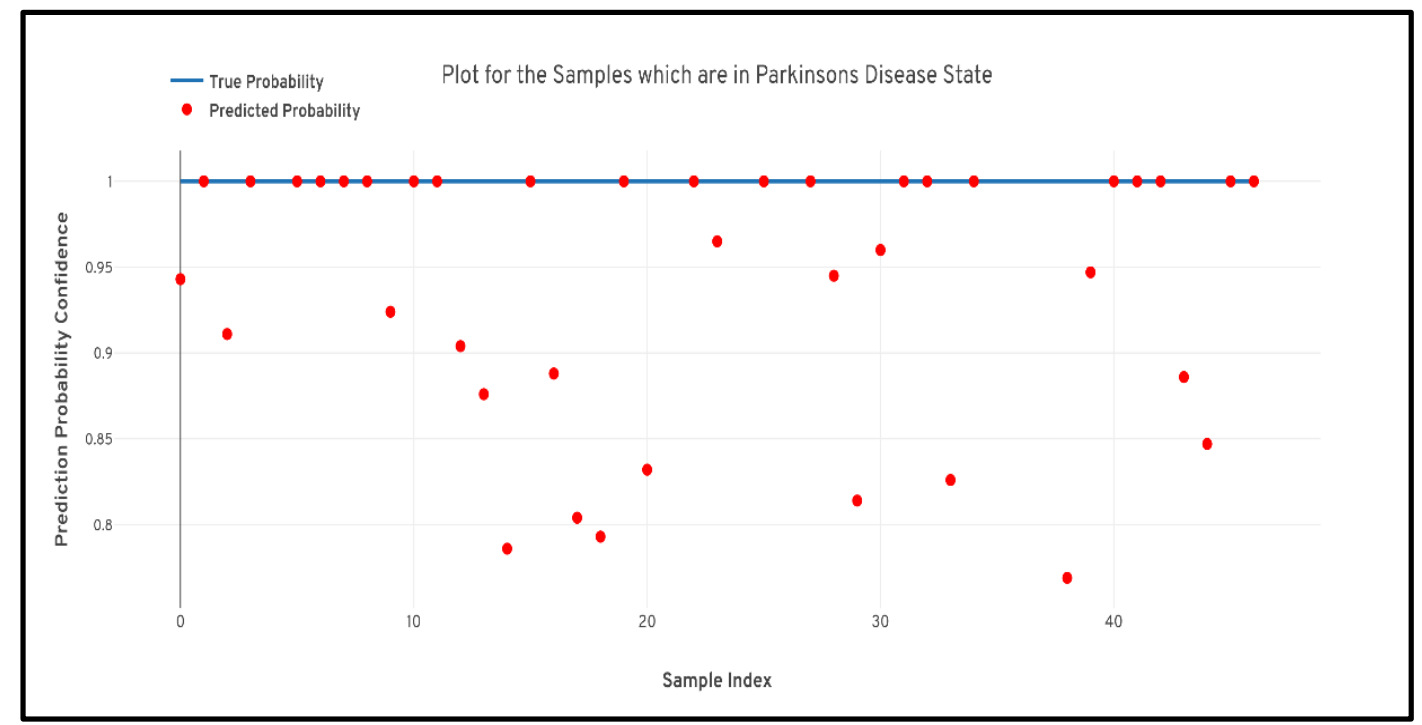

Figure 6. Quantile-quantile plot between the true probability and the prediction probability of the samples belonging to the Parkinson's Disease class. Probabilistic confidence was 0.932 .

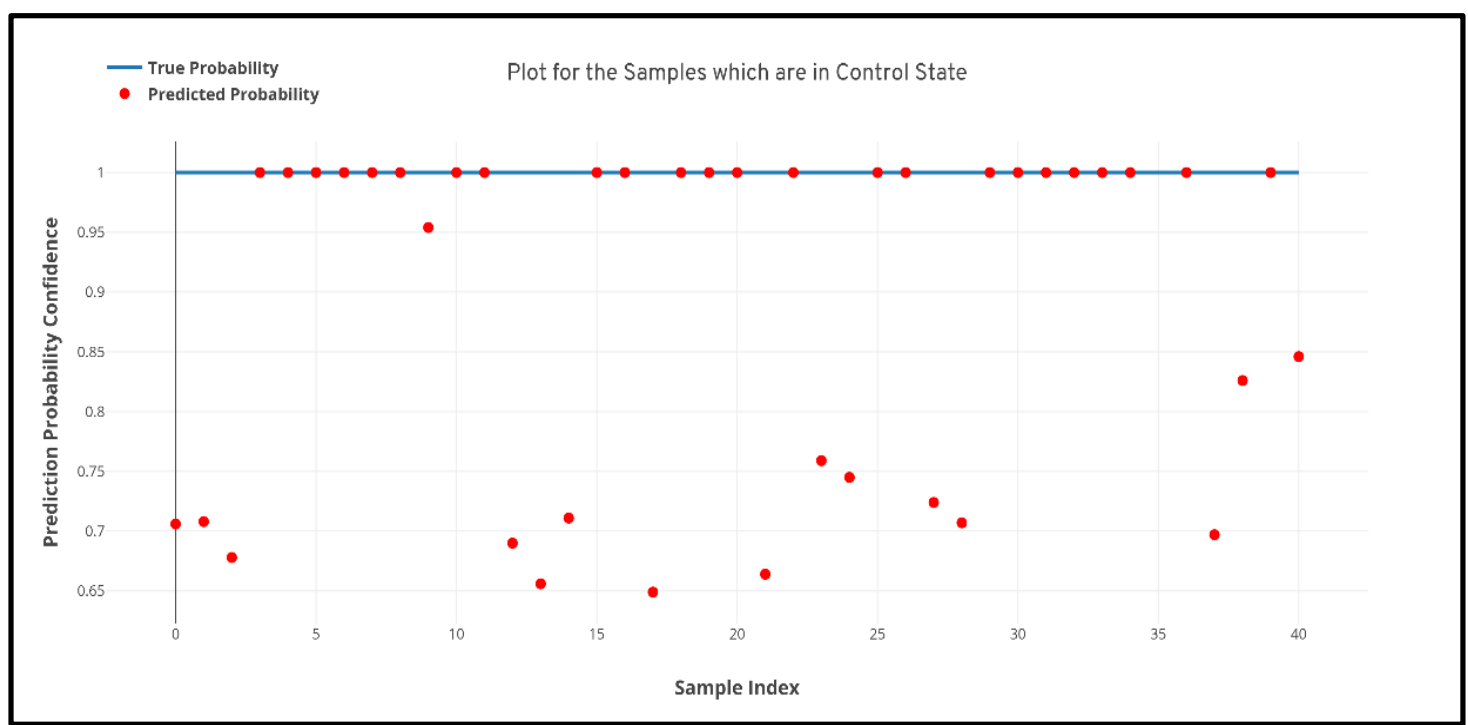

Figure 7. Quantile-quantile plot between the true probability and the prediction probability of the samples belonging to the healthy control class. Probabilistic confidence was 0.843 .

Another very important factor that needs to be measured for evaluating the performance of the deep learning model is the interpretability of the models. The field of healthcare is considered to be a critical field when it comes to the implementation of automated intelligent systems. Therefore, the prime requirement that needs to be provided out of the model is the interpretation behind a particular prediction or cause-effect information that led to a particular prediction. Therefore, to interpret the predictions of the developed 3D CNN model, a class activation map was used [49-51]. Figure 8 shows the class activation map on the sample MRI slices that has been predicted as Parkinson's Disease. The class activation map shows that the model paid much attention to the region of substantia nigra pars compacta (SNc), which is most affected due to the loss of dopaminergic neurons. 


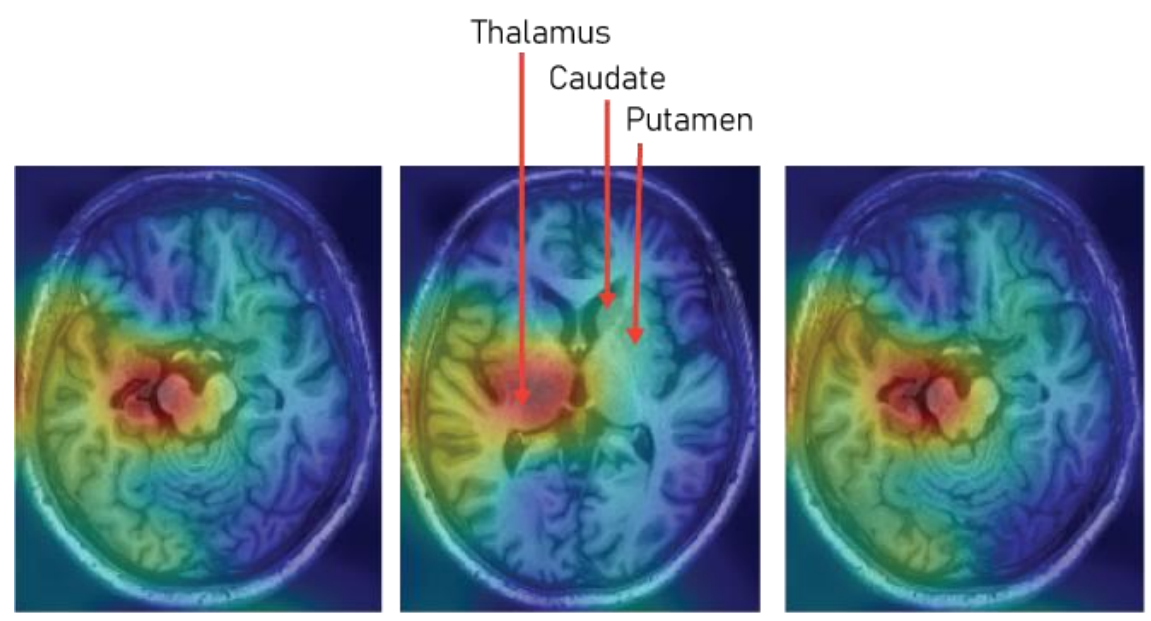

(a)
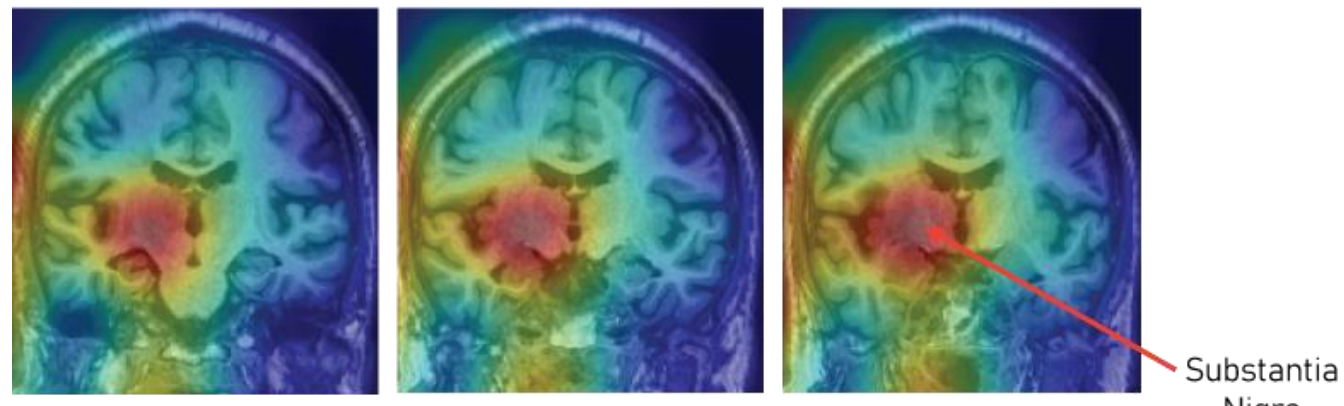

(b)

Nigra
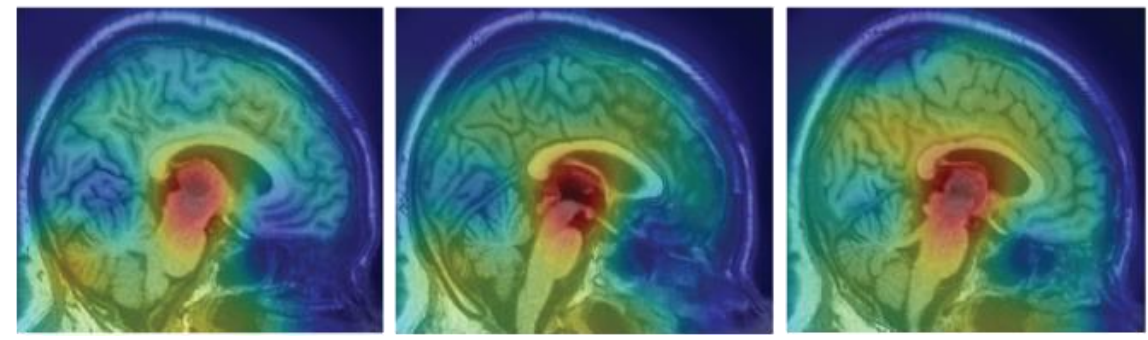

(c)

Figure 8. Class activation maps for sample slices of MRI scan that has been predicted as Parkinson's Disease. (a) Axial view, (b) coronal view, and (c) sagittal view.

\section{Discussion}

The study concerns the development of a 3D convolutional neural network architecture for the detection of Parkinson's Disease from 3T-T1 weighted MRI scans. MRI scans were collected from the PPMI database from two different research groups namely, healthy control and Parkinson's Disease. As discussed, the PPMI is a multicenter study, therefore, the acquired MRI scans had spatial and temporal differences. Therefore, to bring all the MRI scans to the same space, an image registration routine was performed over all the MRI scans. The registration of images was performed using MNIPD2-T1MPRAGE-1 mm atlas. Following registration of the brain MRI scans, a 3D convolutional neural network was developed for the learning intricate patterns in the MRI scans for the detection of Parkinson's Disease and classifying MRI scans into healthy control and Parkinson's Disease categories, respectively.

Before the development of the model, a hypothesis was designed to evaluate the performance metrics of the model. The hypothesis stated that the recall of the Parkinson's Disease class or the true negatives must be more than 0.95 and the probability factor of the mispredicted samples or the false 
negatives must not be more than $60 \%$, and the recall of the healthy class or the true positives must be more than $85 \%$ and the probability factor of the mispredicted samples or the false positives must not be more than $65 \%$. Therefore, to satisfy the prior hypothesis five performance metrics namely, confusion matrix, accuracy, precision, recall, and f1-score, were evaluated. From the results, it can be observed that the model predicted superiorly by plotting a maximum accuracy of $95.29 \%$, recall of 0.943 , the precision of 0.927 , and an $\mathrm{f1}$-score of 0.936 for both the classes. Additionally, for understanding the generalizing capability of the model, 5-split cross-validation was performed and it was observed that the model performed constantly over all the cross-validation splits.

Further to prove the prior hypothesis of the work, a confusion matrix and quantile-quantile plots were depicted in the work, which completely aligned with the hypothesis. In the confusion matrix, it can be observed that there was no misprediction for MRI scans belonging to Parkinson's Disease class. Moreover, from the quantile-quantile plot, it can be observed that all the correctly predicted samples had the prediction confidence of more than $85 \%$ for the MRI scans belonging to the Parkinson's Disease class. Therefore, it can be observed from the results that the developed 3D CNN model performed robustly by plotting superior performance results by completely aligning with the prior hypothesis of the work, and also the model demonstrated a high generalizing capability based on the cross-validation results.

Presently, as the research area in artificial intelligence, machine learning, and deep learning are focused on the interpretability of the black box models. Therefore, to determine and understand whether the model is choosing the correct areas or the regions that are responsible for the detection of Parkinson's Disease, a 3D class activation map (3D CAM) was developed. From the 3D CAM plot depicted in Figure 8, it can be observed that the model has predicted a particular MRI scans as Parkinson's Disease by paying much attention to the substantia nigra region (SNc). Therefore, it can be considered that the model understood the particular areas in the MRI scans which are responsible for Parkinson's Disease.

\section{Conclusions}

In the proposed study, a 3D MRI analysis was performed for the detection of Parkinson's Disease using 3D convolutional neural network. The study leveraged full brain 3D MRI scans to understand intricate patterns in all the subcortical structures of the brain for the detection of Parkinson's Disease. For the evaluation of the CNN model, certain performance metrics were considered, and to validate the values of the performance metrics a prior hypothesis was designed. After, the training of the 3D CNN model it was observed that the model performed superiorly by closely aligning with the prior hypothesis of the study and also demonstrated pretty astounding results. The model developed in the study plotted an overall accuracy of $95.29 \%$, average recall of 0.943 , average precision of 0.927 , and f1-score of 0.936 for both the classes. Moreover, the interpretation of the model over the MRI scans was also evaluated using 3D class activation maps, and it was found that the model paid maximum attention to the substantia nigra region (SNc) for predicting a particular MRI scan as Parkinson's Disease.

To conclude, the outcome of the proposed study is very motivating. However, there remains a huge scope of untouched study concerning the development of innovative architectures that can be leveraged for the detection of Parkinson's Disease using 3D CNN. Moreover, presently the study focused on whole-brain MRI scans, but in the future, it is highly recommended to perform such research by considering specific subcortical structures and the development of more efficient architectures for the detection of Parkinson's Disease.

Author Contributions: Conceptualization was done by S.C. and S.A.; methodology was done by S.C. and S.A.; validation was done by S.C., S.A., and H.-C.K.; formal analysis was done by S.C., S.A., and H.-C.K.; writing original draft was done by S.C. and S.A.; writing-original draft preparation, S.A. and S.C.; writing-review and editing, S.A. and S.C.; supervision, H.-C.K.; project administration, H.-C.K.; funding acquisition, H.-C.K. All authors have read and agreed to the published version of the manuscript. 
Funding: This research was funded by Basic Science Research Program through the National Research Foundation of Korea (NRF), supported by the Ministry of Science, ICT \& Future Planning (NRF-2017R1D1A3B04032905).

Conflicts of Interest: The authors declare no conflict of interest.

\section{References}

1. Davie, C.A. A review of Parkinson's disease. Br. Med. Bull. 2008, 86, 109-127. [CrossRef] [PubMed]

2. Aich, S.; Joo, M.I.; Kim, H.C.; Park, J. Improvisation of classification performance based on feature optimization for differentiation of Parkinson's disease from other neurological diseases using gait characteristics. Int. J. Electr. Comput. Eng. 2019, 9, 5176-5184. [CrossRef]

3. Aich, S.; Pradhan, P.; Park, J.; Sethi, N.; Vathsa, V.; Kim, H.C. A validation study of freezing of gait (FoG) detection and machine-learning-based FoG prediction using estimated gait characteristics with a wearable accelerometer. Sensors 2018, 18, 3287. [CrossRef]

4. Chakraborty, S.; Aich, S.; Kim, H.C. 3D Textural, Morphological and Statistical Analysis of Voxel of Interests in 3T MRI Scans for the Detection of Parkinson's Disease Using Artificial Neural Networks. In Healthcare; Multidisciplinary Digital Publishing Institute: Basel, Switzerland, 2020; Volume 8, p. 34.

5. Aich, S.; Pradhan, P.M.; Chakraborty, S.; Kim, H.C.; Kim, H.T.; Lee, H.G.; Kim, I.H.; Joo, M.I.; Jong Seong, S.; Park, J. Design of a Machine Learning-Assisted Wearable Accelerometer-Based Automated System for Studying the Effect of Dopaminergic Medicine on Gait Characteristics of Parkinson's Patients. J. Healthc. Eng. 2020, 2020, 1823268. [CrossRef] [PubMed]

6. Bakator, M.; Radosav, D. Deep learning and medical diagnosis: A review of literature. Multimodal Technol. Interact. 2018, 2, 47. [CrossRef]

7. Lundervold, A.S.; Lundervold, A. An overview of deep learning in medical imaging focusing on MRI. Zeitschrift für Medizinische Physik 2019, 29, 102-127. [CrossRef] [PubMed]

8. Castellano, G.; Bonilha, L.; Li, L.M.; Cendes, F. Texture analysis of medical images. Clin. Radiol. 2004, 59, 1061-1069. [CrossRef]

9. Shen, D.; Wu, G.; Suk, H.I. Deep Learning in Medical Image Analysis. Annu. Rev. Biomed. Eng. 2017, 19, 221-248. [CrossRef]

10. Esteva, A.; Kuprel, B.; Novoa, R.A.; Ko, J.; Swetter, S.M.; Blau, H.M.; Thrun, S. Dermatologist-level classification of skin cancer with deep neural networks. Nature 2017, 542, 115-118. [CrossRef]

11. Vieira, S.; Pinaya, W.H.; Mechelli, A. Using deep learning to investigate the neuroimaging correlates of psychiatric and neurological disorders: Methods and applications. Neurosci. Biobehav. Rev. 2017, 74, 58-75. [CrossRef]

12. Xiong, H.Y.; Alipanahi, B.; Lee, L.J.; Bretschneider, H.; Merico, D.; Yuen, R.K.; Hua, Y.; Gueroussov, S.; Najafabadi, H.S.; Hughes, T.R.; et al. The human splicing code reveals new insights into the genetic determinants of disease. Science 2015, 347, 1254806. [CrossRef] [PubMed]

13. LeCun, Y.; Bengio, Y.; Hinton, G. Deep learning. Nature 2015, 521, 436-444. [CrossRef] [PubMed]

14. Payan, A.; Montana, G. Predicting Alzheimer's disease: A neuroimaging study with 3D convolutional neural networks. arXiv 2015, arXiv:1502.02506.

15. Hosseini-Asl, E.; Gimel'farb, G.; El-Baz, A. Alzheimer's disease diagnostics by a deeply supervised adaptable 3D convolutional network. arXiv 2016, arXiv:1607.00556.

16. Sarraf, S.; Tofighi, G.; For the Alzheimer's Disease Neuroimaging Initiative. DeepAD: Alzheimer's Disease Classification via Deep Convolutional Neural Networks Using MRI and fMRI. 2016. Available online: HTTPS://WWW.BIORXIV.ORG/CONTENT/EARLY/2016/08/21/070441 (accessed on 15 February 2020).

17. Suk, H.I.; Lee, S.W.; Shen, D.; Alzheimer's Disease Neuroimaging Initiative. Deep ensemble learning of sparse regression models for brain disease diagnosis. Med. Image Anal. 2017, 37, 101-113. [CrossRef] [PubMed]

18. Lin, W.; Tong, T.; Gao, Q.; Guo, D.; Du, X.; Yang, Y.; Guo, G.; Xiao, M.; Du, M.; Qu, X.; et al. Convolutional neural networks-based MRI image analysis for the Alzheimer's disease prediction from mild cognitive impairment. Front. Neurosci. 2018, 12, 777. [CrossRef] [PubMed]

19. Wen, J.; Thibeau-Sutre, E.; Samper-Gonzalez, J.; Routier, A.; Bottani, S.; Durrleman, S.; Burgos, N.; Colliot, O. Convolutional Neural Networks for Classification of Alzheimer's Disease: Overview and Reproducible Evaluation. arXiv 2019, arXiv:1904.07773. 
20. Oh, K.; Chung, Y.C.; Kim, K.W.; Kim, W.S.; Oh, I.S. Classification and Visualization of Alzheimer's Disease using Volumetric Convolutional Neural Network and Transfer Learning. Sci. Rep. 2019, 9, 1-16. [CrossRef]

21. Li, G.; Zhai, G.; Zhao, X.; An, H.; Spincemaille, P.; Gillen, K.M.; Ku, Y.; Wang, Y.; Huang, D.; Li, J. 3D texture analyses within the substantia nigra of Parkinson's disease patients on quantitative susceptibility maps and R2* maps. Neuroimage 2019, 188, 465-472. [CrossRef]

22. Sikiö, M.; Holli-Helenius, K.K.; Harrison, L.C.; Ryymin, P.; Ruottinen, H.; Saunamäki, T.; Eskola, H.J.; Elovaara, I.; Dastidar, P. MR image texture in Parkinson's disease: A longitudinal study. Acta Radiol. 2015, 56, 97-104. [CrossRef]

23. Betrouni, N.; Lopes, R.; Defebvre, L.; Leentjens, A.F.; Dujardin, K. Texture features of magnetic resonance images: A marker of slight cognitive deficits in Parkinson's disease. Mov. Disord. 2020, 35, 486-494. [CrossRef] [PubMed]

24. Ortiz, A.; Munilla, J.; Martínez, M.; Gorriz, J.M.; Ramírez, J.; Salas-Gonzalez, D. Parkinson's Disease Detection using isosurfaces-based features and Convolutional Neural Networks. Front. Neuroinform. 2019, $13,48$. [CrossRef] [PubMed]

25. Kollia, I.; Stafylopatis, A.G.; Kollias, S. Predicting Parkinson's disease using latent information extracted from deep neural networks. In Proceedings of the 2019 International Joint Conference on Neural Networks (IJCNN), Budapest, Hungary, 14-19 July 2019; pp. 1-8.

26. Arthur, D.; Vassilvitskii, S. k-means++: The advantages of careful seeding. In Proceedings of the Eighteenth Annual ACM-SIAM Symposium on Discrete Algorithms (SODA'07), New Orleans, LA, USA, 7-9 January 2007; Society for Industrial and Applied Mathematics: Philadelphia, PA, USA, 2007; pp. 1027-1035.

27. Shinde, S.; Prasad, S.; Saboo, Y.; Kaushick, R.; Saini, J.; Pal, P.K.; Ingalhalikar, M. Predictive markers for Parkinson's disease using deep neural nets on neuromelanin sensitive MRI. Neuroimage Clin. 2019, 22, 101748. [CrossRef] [PubMed]

28. Matsuura, K.; Maeda, M.; Yata, K.; Ichiba, Y.; Yamaguchi, T.; Kanamaru, K.; Tomimoto, H. Neuromelanin magnetic resonance imaging in Parkinson's disease and multiple system atrophy. Eur. Neurol. 2013, 70, 70-77. [CrossRef]

29. Ogisu, K.; Kudo, K.; Sasaki, M.; Sakushima, K.; Yabe, I.; Sasaki, H.; Terae, S.; Nakanishi, M.; Shirato, H. 3D neuromelanin-sensitive magnetic resonance imaging with semi-automated volume measurement of the substantia nigra pars compacta for diagnosis of Parkinson's disease. Neuroradiology 2013, 55, 719-724. [CrossRef]

30. Ohtsuka, C.; Sasaki, M.; Konno, K.; Koide, M.; Kato, K.; Takahashi, J.; Takahashi, S.; Kudo, K.; Yamashita, F.; Terayama, Y. Changes in substantia nigra and locus coeruleus in patients with early-stage Parkinson's disease using neuromelanin-sensitive MR imaging. Neurosci. Lett. 2013, 541, 93-98. [CrossRef]

31. Prasad, S.; Stezin, A.; Lenka, A.; George, L.; Saini, J.; Yadav, R.; Pal, P.K. 3D Neuromelanin-sensitive magnetic resonance imaging of the substantia nigra in Parkinson's disease. Eur. J. Neurol. 2018, 25, 680-686. [CrossRef]

32. Sivaranjini, S.; Sujatha, C.M. Deep learning based diagnosis of Parkinson's disease using convolutional neural network. Multimed. Tools Appl. 2019,1-13. [CrossRef]

33. Krizhevsky, A.; Sutskever, I.; Hinton, G.E. Imagenet classification with deep convolutional neural networks. In Advances in Neural Information Processing Systems; Neural Information Processing Systems Foundation, Inc.: La Jolla, CA, USA, 2012; pp. 1097-1105.

34. Shah, P.M.; Zeb, A.; Shafi, U.; Zaidi, S.F.A.; Shah, M.A. Detection of Parkinson Disease in Brain MRI using Convolutional Neural Network. In Proceedings of the 2018 24th International Conference on Automation and Computing (ICAC), Newcastle upon Tyne, UK, 6-7 September 2018; pp. 1-6.

35. Pereira, H.R.; Ferreira, H.A. Classification of Patients with Parkinson's Disease Using Medical Imaging and Artificial Intelligence Algorithms. In Proceedings of the Mediterranean Conference on Medical and Biological Engineering and Computing, Coimbra, Portugal, 26-28 September 2019; Springer: Cham, Switzerland, 2019; pp. 2043-2056.

36. Long, D.; Wang, J.; Xuan, M.; Gu, Q.; Xu, X.; Kong, D.; Zhang, M. Automatic classification of early Parkinson's disease with multi-modal MR imaging. PLOS ONE 2012, 7, e47714. [CrossRef]

37. Fonov, V.; Evans, A.C.; Botteron, K.; Almli, C.R.; Mckinstry, R.C.; Collins, D.L. Unbiased average age-appropriate atlases for pediatric studies. Neuroimage 2011, 54, 313-327. [CrossRef]

38. Fonov, V.S.; Evans, A.C.; McKinstry, R.C.; Almli, C.R.; Collins, D.L. Unbiased nonlinear average age-appropriate brain templates from birth to adulthood. NeuroImage 2009, S102. [CrossRef] 
39. Alemán-Gómez, Y. IBASPM: Toolbox for automatic parcellation of brain structures. In Proceedings of the 12th Annual Meeting of the Organization for Human Brain Mapping, Florence, Italy, 11-15 June 2006.

40. Xiao, Y.; Fonov, V.; Beriault, S.; Subaie, F.A.; Chakravarty, M.M.; Sadikot, A.F.; Bruce Pike, G.; Louis Collins, D. A dataset of multi-contrast population-averaged brain MRI atlases of a Parkinson's disease cohort. Data Brief. 2017, 12, 370-379. [CrossRef] [PubMed]

41. Xiao, Y.; Fonov, V.; Beriault, S.; Subaie, F.A.; Chakravarty, M.M.; Sadikot, A.F.; Bruce Pike, G.; Louis Collins, D. Multi-contrast unbiased MRI atlas of a Parkinson's disease population. Int. J. Comput. Assist. Radiol. Surg. 2015, 10, 329-341. [CrossRef] [PubMed]

42. Xiao, Y.; Beriault, S.; Bruce Pike, G.; Louis Collins, D. Multicontrast multiecho FLASH MRI for targeting the subthalamic nucleus. Magn. Reson. Imaging 2012, 30, 627-640. [CrossRef]

43. Miikkulainen, R.; Liang, J.; Meyerson, E.; Rawal, A.; Fink, D.; Francon, O.; Raju, B.; Shahrzad, H.; Navruzyan, A.; Duffy, N.; et al. Evolving deep neural networks. In Artificial Intelligence in the Age of Neural Networks and Brain Computing; Academic Press: Cambridge, CA, USA, 2019; pp. 293-312.

44. Frazier, P.I. A Tutorial on Bayesian Optimization. 2018. Available online: https://arxiv.org/abs/1807.02811 (accessed on 15 February 2020).

45. Osborne, M.A. Bayesian Gaussian Processes for Sequential Prediction, Optimisation and Quadrature. Ph.D. Thesis, Oxford University, Oxford, UK, 2010.

46. Zeiler, M.D. Adadelta: An adaptive learning rate method. arXiv 2012, arXiv:1212.5701.

47. Janocha, K.; Czarnecki, W.M. On loss functions for deep neural networks in classification. arXiv 2017, arXiv:1702.05659. [CrossRef]

48. Zhang, Z.; Sabuncu, M. Generalized cross entropy loss for training deep neural networks with noisy labels. In Advances in Neural Information Processing Systems; The MIT Press: Cambridge, CA, USA, 2018; pp. 8778-8788.

49. Zhou, B.; Khosla, A.; Lapedriza, A.; Oliva, A.; Torralba, A. Learning Deep Features for Discriminative Localization. In Proceedings of the 2016 IEEE Conference on Computer Vision and Pattern Recognition (CVPR'16), Las Vegas, NV, USA, 27-30 June 2016.

50. Patro, B.N.; Lunayach, M.; Patel, S.; Namboodiri, V.P. U-cam: Visual explanation using uncertainty based class activation maps. In Proceedings of the IEEE International Conference on Computer Vision, Seoul, Korea, 27-28 October 2019; pp. 7444-7453.

51. Selvaraju, R.R.; Cogswell, M.; Das, A.; Vedantam, R.; Parikh, D.; Batra, D. Grad-cam: Visual explanations from deep networks via gradient-based localization. In Proceedings of the IEEE International Conference on Computer Vision, Venice, Italy, 22-29 October 2017; pp. 618-626. 